\title{
Pilot study provides qualitative evidence for intrinsic motivation in schizophrenia spectrum disorders
}

\author{
Marian W. Roman, Lora Humphrey Beebe ${ }^{*}$, Renee Burk \\ University of Tennessee, Knoxville, USA \\ Email: "lbeebe1@utk.edu
}

Received 13 September 2012; revised 12 October 2012; accepted 20 October 2012

\begin{abstract}
Persons with schizophrenia spectrum disorders (SSDs) face multiple health barriers that are improved with exercise, but they seldom exercise due to a variety of barriers, of which amotivation is primary. In the current study, we investigated the subjective experiences of persons with SSDs following a formal exercise program. Twenty seven persons with SSDs who had taken part in a 16-week walking program provided feedback during exit interviews. Our purpose was to ascertain whether comments provided evidence of development of intrinsic motivation as defined by Ryan and Deci's self determination of behavior theory. Our directed content analysis resulted in 5 thematic codes: autonomy, competence, relatedness, health benefits and personal enjoyment. Results highlight the importance of health-related group activities in supporting competence, relatedness and autonomy. Development and testing of interventions to support intrinsic motivation is critical to improving the health of this vulnerable group.
\end{abstract}

Keywords: Motivation; Exercise; Recovery

\section{INTRODUCTION}

Death rates from diabetes, respiratory/cardiovascular, and other obesity related illnesses are significantly higher among the nearly 2 million Americans with schizophrenia spectrum disorders (SSDs = schizophrenia, schizoaffective disorder and schizophreniform disorder) than the general population [1]. The diagnostic overlap between these disorders is well known and includes positive symptoms (hallucinations and delusions) and an increased risk for adverse physical health outcomes.

The most effective medications for managing SSDs (second generation antipsychotics (SGAs) such as clozapine and olanzapine) are associated with weight gain, glucose dysregulation and diabetes [2-4]. Yet, de-

${ }^{*}$ Corresponding author. spite the well-known benefits of exercise and the health dangers associated with obesity, persons with SSDs are less physically active and fit than people with no mental illness or with other mental illnesses [5]. Persons with SSDs contend with multiple exercise barriers, of which amotivation is primary. People with SSDs rarely exercise, their exercise cessation is nearly double that of the general population [6-8], and they cease exercising primarily because of amotivation [9]. For purposes of our work, we define motivation as a desire that energizes behavior and includes internal aspects like values and external aspects like encouragement.

A few mostly quasi-experimental studies using small numbers of SSD subjects consistently document both psychiatric (reduced depression and anxiety [10-13]) and physical (flexibility, weight loss and/or body mass index) health improvements [6-8,14-16] in response to exercise, but investigations were plagued by difficulties. Every investigation reporting attendance or attrition documented significant problems motivating subjects-attrition rates up to $36 \%$ and attendance as low as $23 \%$ $[6-8,13,14,16]$. The single study not reporting health improvements [17] had extremely low attendance (no one attended more than $50 \%$ of sessions) and high attrition (33\% in 10 weeks). These studies demonstrate the necessity of including motivation in examinations of exercise behavior in SSDs.

Only two published studies have described exercise motivation in SSDs. Archie et al. [9] provided free access to a fitness facility to 20 outpatients for 6 months and monitored their exercise. Dropout was $40 \%$ after 4 months, $70 \%$ after 5 months and 90\% after 6 months. These rates compare unfavorably with general population exercise cessation (50\% after 6 months). In this non-intervention study, the main reason given for dropout was lack of motivation. A qualitative investigation [15] used focus groups to explore responses of six persons with schizophrenia to an exercise program offered at a residential unit in Australia. The following four themes were identified during focus groups with exercisers: the program allowed participants to progress at their 
own pace, physical capacity and fitness improvements, positive group dynamics, and future plans for continued exercise.

More studies are needed to understand the role of exercise motivation in SSDs [18], which is critical to developing interventions to enhance exercise participation in this group. In this manuscript, we report results from exit interviews with SSD outpatients after a 16-week group walking program. The walking group was part of a larger intervention study examining exercise self-efficacy that has been reported in detail elsewhere [19]. In this manuscript, we report pilot data from our qualitative analysis examining the exit interview data for concepts theoretically linked to motivation.

\section{METHODS}

\subsection{Participants}

Institutional Review Board (IRB) approvals and appropriate institutional consents were obtained prior to data collection. Inclusion criteria were: 1) a chart diagnosis of schizophrenia or schizoaffective disorder, any subtype, according to the criteria described in the Diagnostic and Statistical Manual for Mental Disorders [1], 2) English speaking, 3) Stable medication regimen (no medication changes within the last month), and 4) medical clearance for moderate exercise in writing from primary care provider. Exclusion criteria were: 1) chart documentation of mental retardation, developmental delay, uncorrected visual or hearing impairments, 2) hospitalization within the past 12 months for angina pectoris, myocardial infarction, or cardiac surgery, 3) congestive heart failure, 4) cardiac pacemaker, 5) heart rate $>100$ or $<50$ at rest, 6) uncontrolled hypertension (blood pressure exceeding 140/90 on 3 consecutive readings despite adequate treatment), 7) history of spinal or hip fractures or hip or knee arthroplasty, and 8) neuromuscular or orthopedic limitations to normal, unassisted ambulation.

The 97 participants in the larger study completed study activities in 5 consecutive cohorts. Participants in cohort one $(n=17)$ were ineligible for exit interviews due to the additional time required to obtain IRB approval to add exit interviews to the previously approved study procedures. The remaining 63 participants who completed the 16 weeks walking program were offered exit interviews. Of that number 27 (43\%) agreed and 36 (57\%) declined. No incentives were available for exit interview participation, and no data were collected regarding reasons for declining exit interviews.

We here report our pilot results from the 27 exit interviews. Participants completing exit interviews ranged in age from 18 to 68 years with an average age of 46.9 years $(\mathrm{SD}=2.0)$. A slight majority were male $(\mathrm{n}=14$, $52 \%$ ) and Caucasian ( $\mathrm{n}=14,52 \%)$. Most were living with family members at the time of the study. All subjects were prescribed antipsychotic medication; the most commonly prescribed medications were oral second generation antipsychotics and antiparkinsonians. See Table 1.

\subsection{Measures}

An exit interview form was used to gather feedback about participant experiences during the group walking program. The form included four open ended questions (See Table 2).

\subsection{Procedure}

Blinded research assistants (RAs) invited participants to provide exit interviews immediately after the final walking group. RAs were graduate students and were trained by L.H.B. before conducting interviews. Exit interviews were conducted in a private room, ranged from 5 to 20 minutes in length and were transcribed verbatim.

\subsection{Qualitative Thematic Analysis}

Directed content analysis is a qualitative method designed "to validate or extend conceptually a theoretical framework or theory" [20]. In contrast to other qualitative methods, directed analysis requires that the theory be identified before data analysis begins. Because exercise motivation was the focus of the larger study, we chose the self determination of behavior theory SDBT [21] to guide our directed content analysis.

SDBT [21-23] posits that motivation (defined as initiation and persistence) is intrinsic and arises from innate energies. The theory proposes that meeting psychological needs enhances the development of intrinsic motivation. Ryan \& Deci [21] stated that intrinsic motivation is enhanced when competence, autonomy and relatedness needs are met. Competence is defined as mastering challenging tasks within one's environment; autonomy as a sense of ownership over one's behaviors; and relatedness as a meaningful connection with others [21]. We used autonomy, competence and relatedness as the starting point for our directed content analysis.

All of the transcribed interviews were coded and analyzed using an Excel spread sheet format. We used the directed content analysis approach [20], using the three theoretical concepts (autonomy, competence and relatedness) as initial codes.

Assuring validity of findings in directed content analysis is a two-step process: developing a coding scheme that is faithful to the theory, and orienting coders to the focal concepts. If the codes match the theoretical definitions, then the coding is regarded as producing valid data [24]. The analysts (R.B. and M.R.) performed an initial cross-coding internal reliability check by pilot 
Table 1. Characteristics of persons with schizophrenia spectrum disorders completing and declining exit interviews $(\mathrm{N}=63)$.

\begin{tabular}{|c|c|c|}
\hline Characteristic & Subjects completing exit interviews $(n=27)$ & Subjects declining exit interviews $(n=36)$ \\
\hline Diagnosis & $\mathrm{n}, \%$ & n, \% \\
\hline Schizoaffective & 18,67 & 28,78 \\
\hline Schizophrenia & 9,33 & 8,22 \\
\hline \multicolumn{3}{|l|}{ Sex } \\
\hline Female & 13,48 & 18,50 \\
\hline Male & 14,52 & 18. 50 \\
\hline \multicolumn{3}{|l|}{ Race } \\
\hline Caucasian & 13,48 & 20,56 \\
\hline African American & 14,52 & 16,44 \\
\hline \multicolumn{3}{|l|}{ Living Arrangement } \\
\hline Alone & 9,33 & 13,36 \\
\hline With family & 12,44 & 17,47 \\
\hline With paid caregiver & 6,23 & 6,17 \\
\hline \multicolumn{3}{|c|}{ Prescribed Medications } \\
\hline Oral SGAs & 20,74 & 26,72 \\
\hline Oral FGAs & 4,15 & 6,17 \\
\hline Depot FGAs & 5,19 & 8,22 \\
\hline Depot SGAs & 5,19 & 5,14 \\
\hline Antidepressants & 15,56 & 19, 53 \\
\hline Mood stabilizers & 7,26 & 14,39 \\
\hline Antianxiety & 4,15 & 12,33 \\
\hline Antiparkinsonian & 2,8 & 0,0 \\
\hline Hypnotics & 5,19 & 4,11 \\
\hline Other $^{\mathrm{a}}$ & 22,81 & 30,83 \\
\hline
\end{tabular}

Notes: SGA = second generation antipsychotic; FGA = first generation antipsychotic; ${ }^{\text {a }}=$ Medications prescribed for physical illnesses, most commonly angiotensin converting enzyme (ACE) inhibitors $(n=18)$ and lipid lowering agents $(n=17)$.

Table 2. Exit interview questions asked of persons with schizophrenia spectrum disorders after group walking program $(\mathrm{N}=27)$.

1. What did you think of the walking groups?

2. Do you have any suggestions for improving the walking groups?

3. Are there any changes we could make that would have made it easier for you to attend the walking group?

4. Is there anything else you would like to share about your experiences in the walking group?

coding 15 responses before beginning formal data analysis. This process is known as seeking an agreement coefficient [25] and contributes to internal validity. Having exceeded $90 \%$ concordance in pilot coding, the text coding commenced with each analyst reading carefully for evidence of autonomy, competence and relatedness. Repetitive words, concepts and phrases were recognized. The coding of each transcript was compared periodically between analysts, and discrepancies were discussed and resolved to ensure consistency.
It is expected in a directed content analysis that some statements will not be categorized within the theoryderived codes; data not categorized within the theoryderived codes are set aside for a second analysis [20]. During review conferences, the two analysts monitored one another to reduce the likelihood of "forcing" data into the three SDBT-derived codes. Items deemed not to match the theory derived codes were analyzed using traditional atheoretical content analysis [26], and the inter rater reliability process was again employed. 


\section{RESULTS}

The subjective responses of our participants to a 16-week exercise program of group walking can by described using the following 5 thematic codes: autonomy, competence, relatedness, health benefits and personal enjoyment. The relatedness, competence and autonomy codes derive from SDBT [21]. Besides the three theory-derived codes, two additional codes were identified-personal enjoyment and health benefits.

Theory-derived themes. The SDBT concept of relatedness (feeling a meaningful connection with others [21] appeared most often in the responses $(\mathrm{n}=33)$. Statements reflecting relatedness included: "I made some friends" "A chance to associate with people" and "Walking with others helped". Competence (effectively mastering challenging tasks within one's environment [21] was evident in 27 statements, such as "I can bend over and tie my shoes without getting winded”, "Built up my breath and endurance", and "Look, see what I can do?" (as a participant demonstrated lower extremity flexibility). Autonomy (perceiving self as source of causality) [21] was evident in 21 items. Examples of such statements included "Plan to keep walking after group is over", "Walking at home (now) and dieting", "Using my exercise tape at home", "Now I'm trying to stop smoking". Autonomy was sometimes linked with competence in a single statement. "Gave me a sense of personal accomplishment", "Walking is helping me be more responsible" and " $\{\mathrm{I}\}$ Liked getting good numbers (on the pedometer) while walking" are examples of statements meeting the criteria for both autonomy and competence categories. As Ryan \& Deci stated, "feelings of competence will not enhance intrinsic motivation unless accompanied by an internal perceived locus of causality" [23]. Although the two concepts in these statements resulted in their receiving double codes, the statements do not attest to the primacy of autonomy, or true self-esteem as was suggested by the theorists' early SDBT research [27]. Relatedness and competence were also paired in several statements, such as "Walking and talking with a group was fun, gave (me) will power to walk" and "If I keep you guys in mind I'll be exercising by myself". The suggested message of internalizing the group expectations in the latter statement is noteworthy in its resonance with the continuum of internalization in the larger SDBT theory [27].

Additional themes. The two themes identified in addition to the theory derived codes comprised personal enjoyment of the walking groups, and the health benefits of walking. From improvement in arthritis, to "I lost two pounds" to "feeling better each day" or "I had fun", the perceived effects on pleasure and health were numerous. Enjoyment and health effects appeared 23 and 31 times respectively.

\section{DISCUSSION}

This manuscript reports pilot data on the subjective experiences of persons with SSDs following a formal exercise program. Our findings echo those of Fogarty \& Happell [15]. For example, our participants reported a sense of accomplishment ("built up my breath and endurance"), similar to that of residential patients with SSDs participating in exercise, who related "it got a lot easier as I went along" [15]. Participants in both studies noted improvement in a number of physical health parameters, expressed positive responses to group dynamics occurring during the exercises, and reported exercising independently outside the study-sponsored programs.

Wilson and colleagues [28] reviewed the use (mainly quantitative) of the SDBT framework in exercise studies and concluded that the available evidence provides strong support for many SDBT propositions. That work has primarily focused on deriving tools to measure SDBT in exercise. Our finding of the three SDBT conceptual components for improving intrinsic motivation (relatedness, autonomy and competence) in exit interviews by exercise participants supports the use of SDBT in SSD samples.

The SDBT concepts of autonomy and competence observed in this dataset correspond to several motivational components identified by others, including self-direction [29] and empowerment [30]; while the relatedness concept corresponds to peer support [31]. Meeting needs for competence, autonomy and relatedness through the provision of health related group activities like those reported here may enhance multiple areas of health and health related behaviors by supporting the development of intrinsic motivation. Our additional concepts of health improvement and enjoyment relate to quality of life improvements noted in the literature [32,33].

These results have numerous implications for the use of concepts of intrinsic motivation to develop theory based interventions for persons with SSDs, as recommended by Yamada [34]. Our subjects reported experiences of relatedness, competence and autonomy [21] during our group exercise program. According to SDBT, experiencing these concepts enhances intrinsic motivetion. Thus, it appears that interventions addressing these components would hold promise for enhancing exercise motivation in persons with SSDs. Clinicians and researchers should consider offering exercise interventions in a group format to support the experience of relatedness. Exercise groups could incorporate dedicated time for social interaction and the provision of peer feedback about progress to enhance feelings of relatedness. The SDBT concept of competence could be fostered by ensuring that exercise interventions include a mechanism for assisting persons with SSDs to identify achievable exercise goals. Highlighting exercise related gains and 
providing positive reinforcement may also foster a sense of competence. Autonomy might be supported by providing participants with tools (such as pedometers) for monitoring their exercise and activity levels outside the formal exercise program.

There are several reasons our results must be viewed with caution. Of 63 participants who completed the walking program in the larger study, 27 provided exit interviews. Since exit interviews were offered only at the conclusion of the 16-week walking program, the findings reported here do not represent participants who ceased walking before 16 weeks. Nevertheless, it is noteworthy that those who chose to engage in exit interviews referenced positive feelings about self and relatedness, both of which are often problematic for persons with SSDs.

\section{CONCLUSION}

In conclusion, this pilot study suggests that persons with SSDs experience a variety of positive responses to exercise intervention. The themes identified in their responses correspond to concepts from SDBT [21,23] and support viewing motivation as intrinsic. Our findings provide evidence for Ryan \& Deci's [21,23] view of motivation as an intrinsic characteristic that is enhanced when competence, autonomy and relatedness needs are met. Designing interventions using SDBT components may help address motivation barriers and increase exercise behavior in persons with SSDs, resulting in multiple health benefits.

\section{ACKNOWLEDGEMENTS}

This study was supported by a grant from the National Institutes of Mental Health, project number 1R03MH79047-02.

\section{REFERENCES}

[1] American Psychiatric Association (APA) (2000) Diagnostic and statistical manual of mental disorders. 4th Edition, American Psychiatric Association, Washington DC.

[2] Ananth, J., Venkatesh, R., Burgoyne, K., Gadasalli, R., Binford, R. and Gunatilake, S. (2004) Atypical antipsychotic induced weight gain: Pathophysiology and management. Annals of Clinical Psychiatry, 16, 75-85.

[3] Jin, H., Meyer, J.M. and Jeste, D.V. (2004) Atypical antipsychotics and glucose dysregulation: A systematic review. Schizophrenia Research, 71, 195-212. doi:10.1016/j.schres.2004.03.024

[4] Newcomer, J.W. (2004) Abnormalities of glucose metabolism associated with atypical antipsychotic drugs. Journal of Clinical Psychiatry, 65, 36-46.

[5] Beebe, L.H. (2005) Effects of exercise on mental and physical health parameters of persons with schizophrenia. Issues in Mental Health Nursing, 26, 661-676. doi:10.1080/01612840590959551
[6] Centorrino, F., Wurtman, J.J., Duca, K.A., Fellman, V.H., Fogarty, K.V., Berry, J.M., Guay, D.M., Romeling, M., Kidwell, J., Cincotta, C.L. and Baldessarini, R.J. (2006) Weight loss in overweight patientsmaintained on atypical antipsychotic agents. International Journal of Obesity, 30, 1011-1016. doi:10.1038/sj.ijo.0803222

[7] Kwon, J.S., Choi, J.S., Bahk, W.M., Kim, C.Y., Kim, C.H., Shin, Y.C., Park, B.J. and Oh, C.G. (2006) Weight management program for treatment-emergent weight gain in Olanzapine-treated patients with schizophrenia or schizoaffective disorder: A 12-week randomized controlled clinical trial. Journal of Clinical Psychiatry, 67, 547-553.

[8] Pendlebury, J., Haddad, P. and Dursun, S. (2005) Evaluation of a behavioral weight managementprogramme for patients with severe mental illness: 3 year results. Human Psychopharmacology, 20, 447-448.

[9] Archie, W., Wilson, J.H., Osborne, S., Hobbs, H. and McNiven, J. (2003) Pilot study: Access to fitness facility and exercise levels in olanzapine-treated patients. Canadian Journal of Psychiatry, 48, 628-632.

[10] Acil, A.A., Dogan, S. and Dogan, O. (2008) The effects of physical exercises to mental state and quality of life in patients with schizophrenia. Journal of Psychiatric and Mental Health Nursing, 15, 808-815. doi:10.1111/j.1365-2850.2008.01317.x

[11] Chamove, A.S. (1986) Positive short-term effects of activity on behavior in chronic schizophrenic patients. British Journal of Clinical Psychology, 25, 125-133. doi:10.1111/j.2044-8260.1986.tb00681.x

[12] Gimino, F.A. and Levin, S.J. (1984) The effects of aerobic exercise on perceived self-image in post-hospitalized schizophrenic patients. Medicine \& Science in Sports \& Exercise, 16, 139.

[13] Pelham, T.W., Campagna, P.D., Ritvo, P.G. and Birnie, W.A. (1993) The effects of exercise therapy on clients in a psychiatric rehabilitation program. Psychosocial Rehabilitation Journal, 16, 75-84.

[14] Chen, C.K., Chen, Y.C. and Huang, Y.S. (2009) Effects of a 10 -week weight control program on obese patients with schizophrenia or schizoaffective disorder: A 12 month follow up. Psychiatry and Clinical Neurosciences, 63, 17-22.

[15] Fogarty, M., Happell, B. and Pininkahana, J. (2004) The benefits of an exercise program for people with schizophrenia: A pilot study. Psychosocial Rehabilitation Journal, 28, 173-176.

[16] Vreeland, B., Minsk, S., Menza, M., Rigussio-Radler, D., Roemheld-Hamm, B. and Stern, R. (2003) A program for managing weight gain associated with atypical antipsychotics. Psychiatric Services, 54, 1155-1157. doi:10.1176/appi.ps.54.8.1155

[17] Ball, M.P., Coons, V.B. and Buchanan, R.W. (2001) A program for treating olanzapine related weight gain. Psychiatric Services, 52, 967-969. doi:10.1176/appi.ps.52.7.967

[18] Roberts, S.H. and Bailey, J.E. (2011) Incentives and barriers to lifestyle interventions for people with serious 
mental illness: A narrative synthesis of quantitative, qualitative and mixed method studies. Journal of Advanced Nursing, 67, 690-708.

[19] Beebe, L.H. et al. (2011) Effect of a motivational intervention on exercise behavior in persons with schizophrenia spectrum disorders. Community Mental Health Journal, 47, 628-636.

[20] Hsieh, H.F., Shannon, S.E. (2005) Three approaches to qualitative content analysis. Qualitative Health Research, 15, 1277-1288. doi:10.1177/1049732305276687

[21] Ryan, R.M., Deci, E.L. (2002) Overview of self-determination theory: An organismic dialectical perspective. In: Deci, E.L. and Ryan, R.M., Eds., Handbook of SelfDetermination Research, The University of Rochester Press, Rochester, 6-33.

[22] Deci, E. and Ryan, R.M. (1985) Intrinsic motivation and self-determination in human behavior. Plenum Press, New York.

[23] Ryan, R.M. and Deci, E.L. (2000) Self-determination theory and the facilitation of intrinsic motivation, social development and well-being. American Psychologist, 55, 68-78.

[24] Potter, W.J. and Levine-Donnerstein, D. (1999) Rethinking validity and reliability in content analysis. Journal of Applied Communication research, 27, 258-284. doi:10.1080/00909889909365539

[25] Weber, R.P. (1990) Basic content analysis. 2nd Edition, Sage, Newbury Park.

[26] Lindseth, A. and Norberg, A. (2004) A phenomenological hermeneutical method for researching lived experience. Scandinavian Journal of Caring Sciences, 18, 145-153. doi:10.1111/j.1471-6712.2004.00258.x

[27] Ryan, R.M. (1982) Control and information in the intrapersonal sphere: An extension of cognitive evaluation theory. Journal of Personality and Social Psychology, 43, 450-461.

[28] Wilson, P.M., Mack, D.E. and Grattan, K.P. (2008) Understanding motivation for exercise: A Self-determination theory perspective, Canadian Psychology, 49, 250256.

[29] Heiss, C., Ziegler, M., Engbert, K., Gropel, P. and Brand, R. (2010) Self-leadership and volition: Distinct and potentially supplemental constructs? Psychological Reports, 107, 447-462.

doi:10.2466/01.03.07.14.PR0.107.5.447-462

[30] Ishikawa, H. and Kiuchi, T. (2010) Health literacy and health communication. Biopsychosocial Medicine, 5, 418.

[31] Murray, N.J., Gasper, A.V., Irvine, L., Scarpello, T.J. and Sampson, M.J. (2012) A motivational peer support program for type 2 diabetes prevention delivered by people with type 2 diabetes: The UEA-IFG feasibility study. The Diabetes Educator (in press).

[32] Gorczynski, P. and Faulkner, G. (2011) Exercise therapy for schizophrenia. Schizophrenia Bulletin, 36, 665-666. doi:10.1093/schbul/sbq049

[33] Vancampfort, D., Vansteelandt, K., Scheewe, T., Probst, M., Knapen, J., DeHerdt, A. and DeHert, M. (2012) Yoga in schizophrenia: A systematic review of randomized controlled trials. Acta Psychiatrica Scandinavica (in press). doi:10.1111/j.1600-0447.2012.01865.X

[34] Yamada, A., Lee, K.K., Dinh, T.Q., Barrio, C. and Brekke, J.S. (2010) Intrinsic motivation as a mediator of relationships between symptoms and functioning among individuals with Schizophrenia spectrum disorders in a diverse urban community. The Journal of Nervous and Mental Disease, 198, 28-34. doi:10.1097/NMD.0b013e3181c8aa71 